\title{
Influência do balanço cátion-aniônico da dieta no rúmen e no desempenho de ovinos
}

[Influence of dietary cation-anion balance on rumen and performance variables in sheep]

\author{
G.R. Del Claro ${ }^{1}$, M.A. Zanetti ${ }^{2}$, F.A. Paiva ${ }^{1}$, A. Saran Netto ${ }^{1}$, \\ M.S.V. Salles ${ }^{2}$, L.B. Correa ${ }^{1}$ \\ ${ }^{1}$ Estudante de Pós-Graduação - FZEA - USP - Pirassununga \\ ${ }^{2}$ Faculdade de Zootecnia e Engenharia de Alimentos - Universidade de São Paulo \\ Rua Duque de Caxias Norte, 225 \\ 13630-000 - Pirassununga, SP
}

\begin{abstract}
RESUMO
Estudou-se o efeito do balanço cátion-aniônico da dieta (BCAD) no desempenho animal e na fermentação ruminal, utilizando-se 25 carneiros machos, da raça Santa Inês, durante 75 dias. O delineamento experimental foi em blocos ao acaso, com cinco tratamentos. Para a manipulação do BCAD, foram adicionados sulfato de amônio e bicarbonato de sódio, obtendo-se os seguintes tratamentos: $-160,-40$, 140,250 e $500 \mathrm{mEq} / \mathrm{kg}$ de matéria seca. O aumento do BCAD resultou em aumentos da ingestão de matéria seca, do ganho diário, da eficiência alimentar e do $\mathrm{pH}$ ruminal $(\mathrm{P}<0,05)$. O perfil de ácidos graxos ruminais não foi afetado pelo BCAD.
\end{abstract}

Palavras-chave: ovino, balanço cátion-aniônico, rúmen, matéria seca, $\mathrm{pH}$

\begin{abstract}
The effect of dietary cation-anion balance (DCAB) on dry matter intake, daily weight gain, feed conversion efficiency, rumen $\mathrm{pH}$ and rumen volatile fatty acid profile in Santa Ines sheep was studied. Dietary treatments were formulated combining sodium sulphate and sodium bicarbonate in appropriate ratios to achieve DCAB balance of $-160,-40,140,250$ and $500 \mathrm{mEq} / \mathrm{kg}$ dry matter. Twenty five male lambs were assigned to the five treatments in a randomized block design for a 75-day feeding trial. Increasing DCAB was associated with increasing voluntary dry matter intake, daily weight gain, feed conversion efficiency and rumen $\mathrm{pH}(\mathrm{P}<0.05)$; but DCAB did not affect the volatile fatty acid profile in the rumen.
\end{abstract}

Keywords: sheep, rumen, cation-anion balance, dry matter intake, $\mathrm{pH}$

\section{INTRODUÇÃO}

O balanço cátion-aniônico dietético (BCAD), também conhecido por diferença cátion-aniônica da dieta (DCAD), balanço eletrolítico (BE) ou balanço iônico da dieta (BID), representa a diferença entre os cátions e os ânions fixos totais, presentes na dieta. A maioria dos experimentos calcula o BCAD em mEq de $\left(\mathrm{Na}^{+}\right.$
$\left.+\mathrm{K}^{+}\right)-\left(\mathrm{Cl}^{-}+\mathrm{SO}_{4}{ }^{-}\right)$por $\mathrm{kg}$ ou 100 gramas de matéria seca. $\mathrm{O}$ enxofre, apesar de não ser um íon fixo, é incluído no cálculo do BCAD para ruminantes, pois os sulfatos acidificam diretamente os fluidos biológicos. Esses íons são escolhidos para o cálculo do BCAD por influenciarem o balanço ácido-base apesar de terem influência em outras funções no metabolismo animal (Block, 1994).

Projeto financiado pela FAPESP

Recebido para publicação em 1 de dezembro de 2003

Recebido para publicação, após modificações, em 3 de janeiro de 2005

* Autor para correspondência (corresponding author)

E-mail: gdelclaro@yahoo.com.br 
A homeostase ácido-base é um fator de fundamental importância em qualquer espécie animal. Para que os processos vitais transcorram normalmente, o $\mathrm{pH}$ dos fluidos corporais deve ser mantido entre limites estritos. Pequenas modificações nessa concentração podem causar alterações na velocidade das reações químicas das células. Deve-se corrigir o acréscimo de ácido, ou íon hidrogênio, aos líquidos corporais, já que com o metabolismo há produção constante de ácidos. Em condições normais, há um contínuo acréscimo de ácidos aos líquidos corporais, pela ingestão ou pelo metabolismo celular (Cunninghan, 1999).

O equilíbrio ácido-base tem influência direta no metabolismo animal e, por isso, uma relação intrínseca com desempenho (Miles e Butcher, 1993). Um fator que poderia explicar a relação entre o BCAD e o desempenho é a bomba de $\mathrm{Na}^{+}$ e $\mathrm{K}^{+}$. Esse processo é responsável pelo consumo de aproximadamente $40 \%$ da energia de manutenção (Block, 1994). Alterações promovidas pelo BCAD na fermentação ruminal também poderiam explicar a sua relação com o desempenho. Tucker et al. (1988), ao arraçoarem vacas holandesas em lactação, com dietas contendo diferentes níveis de $\mathrm{BCAD}$, concluíram que o $\mathrm{pH}$ do líquido ruminal diminuiu com o decréscimo do balanço cátion-aniônico, mas não houve alteração na fermentação ruminal. Vagnoni e Oetzel (1998), ao trabalharem com vacas secas, encontraram decréscimo no $\mathrm{pH}$ do líquido ruminal quando da utilização de dietas aniônicas, entretanto a fermentação ruminal não foi alterada.

Vacas em lactação devem ter o BCAD altamente positivo, já que esses ruminantes apresentam altas taxas metabólicas e, portanto, há uma tendência para o ambiente celular tornar-se ácido (Block, 1994). Tucker et al. (1988) sugeriram em vacas lactantes, para o máximo desempenho, que o BCAD esteja entre +200 a $+375 \mathrm{mEq} / \mathrm{kg}$ de matéria seca (MS). Den Hartog et al. (1990) relataram desempenho inferior de vitelos com $\mathrm{NaHCO}_{3}$ e $\mathrm{BCAD}$ de $+200 \mathrm{mEq} / \mathrm{kg}$ de $\mathrm{MS}$ em relação ao grupo-controle, enquanto o grupo com $\left(\mathrm{NH}_{4}\right) \mathrm{SO}_{4}$ e $\mathrm{BCAD}$ de $-100 \mathrm{mEq} / \mathrm{kg}$ de $\mathrm{MS}$ apresentou resultados intermediários. Para bovinos de corte, Ross et al. (1994), ao trabalharem com novilhos Angus em crescimento, concluíram que o melhor desempenho foi conseguido com BCAD de 150 a $300 \mathrm{mEq}$ de $(\mathrm{Na}+\mathrm{K})-(\mathrm{Cl}) / \mathrm{kg}$ de MS. Fauchon et al. (1995) observaram que dietas entre $500 \mathrm{e}$ $700 \mathrm{mEq}$ de $(\mathrm{Na}+\mathrm{K})-(\mathrm{Cl}) / \mathrm{kg}$ de MS foram as que apresentaram maior desempenho para ovinos em crescimento. Jackson et al. (2001), em experimento conduzido com bezerros tratados com BCAD de 0 ou $200 \mathrm{mEq}[(\mathrm{Na}+\mathrm{K})-$ $(\mathrm{Cl}+\mathrm{S})] / \mathrm{kg}$ de $\mathrm{MS}$, não observaram diferenças no desempenho entre os dois tratamentos.

O trabalho teve por objetivo verificar os efeitos de diferentes relações entre cátions e ânions sobre a ingestão de matéria seca, ganho de peso diário, eficiência alimentar e fermentação ruminal em ovinos da raça Santa Inês.

\section{MATERIAL E MÉTODOS}

Utilizaram-se 25 cordeiros machos, da raça Santa Inês, alojados em gaiolas de estudo de metabolismo, equipadas com bebedouro automático, cocho e coletor de urina.

Os animais foram alimentados com dieta calculada para suprir suas necessidades, segundo o Nutrient... (1985). Foram oferecidos cinco tratamentos, diferindo nas concentrações de bicarbonato de sódio, sulfato de amônio e caulim em cinco níveis de BCAD $(-160 ;-40 ; 140 ; 260$; $500 \mathrm{mEq} / \mathrm{kg}$ de MS). O tratamento com $140 \mathrm{mEq} / \mathrm{kg}$ de MS foi considerado o controle, pois continha os níveis de $\mathrm{Na}^{+} ; \mathrm{K}^{++} ; \mathrm{Cl}^{-}$e $\mathrm{S}^{-2}$ preconizados pelo Nutrient... (1985) para ovinos. Nas Tab. 1 e 2 apresentam-se a proporção dos ingredientes e a composição química bromatológica das dietas experimentais.

O experimento teve duração de 90 dias, sendo os 15 primeiros usados para adaptação à ração total. Inicialmente os animais foram pesados, desverminados e distribuídos em gaiolas de estudo de metabolismo. A água foi fornecida à vontade por sistema de abastecimento contínuo. 


\section{Del Claro et al.}

Tabela 1. Composição percentual dos ingredientes das dietas em base seca e balanço cátion-aniônico $(\mathrm{BCAD})$ da dieta em $\mathrm{mEq}$ de $\left\{(\mathrm{Na}+\mathrm{K})-\left(\mathrm{Cl}+\mathrm{SO}_{4}\right)\right\}$

\begin{tabular}{lccccc}
\hline Ingrediente & $\mathrm{N} 1$ & $\mathrm{~N} 2$ & $\mathrm{C}$ & $\mathrm{P} 1$ & $\mathrm{P} 2$ \\
\hline Casca de algodão & 30,00 & 30,00 & 30,00 & 30,00 & 30,00 \\
Milho & 43,43 & 43,43 & 43,43 & 43,43 & 43,43 \\
Soja extrusada & 20,72 & 20,72 & 20,72 & 20,72 & 20,72 \\
Calcário & 1,35 & 1,35 & 1,35 & 1,35 & 1,35 \\
Suplemento mineral* & 0,50 & 0,50 & 0,50 & 0,50 & 0,50 \\
Caulim & 2,00 & 2,40 & 3,07 & 2,07 & - \\
$\mathrm{NaHCO}_{3}$ & - & - & - & 1,00 & 3,07 \\
$\mathrm{NH}_{3} \mathrm{SO}_{4}$ & 2,00 & 1,20 & - & - & - \\
Uréia & 0,00 & 0,40 & 0,93 & 0,93 & 0,93 \\
BCAD & -160 & -40 & 140 & 250 & 500 \\
\hline
\end{tabular}

(*)500g de suplemento mineral contêm: 418,0g de cloreto de sódio; $14,3 \mathrm{~g}$ de sulfato de ferro II; $11,5 \mathrm{~g}$ de sulfato de manganês II; $5,6 \mathrm{~g}$ de óxido de zinco; $0,32 \mathrm{~g}$ de iodato de sódio; $6,4 \mathrm{~g}$ de sulfato de cobre II e $0,2 \mathrm{~g}$ de sulfato de cobalto; $0,08 \mathrm{~g}$ de selenito de sódio; $40 \mathrm{~g}$ de flor-de-enxofre; $0,09 \mathrm{~g}$ selenito de sódio. $3,34 \mathrm{~g}$ de complexo vitamínico com vitamina $\mathrm{A}$ (palmitato), vitamina $\mathrm{D}_{3}$ e tocoferol (acetato). MS = matéria seca. N1, N2, C, P1 e P2 = tratamentos segundo o BCAD.

Tabela 2. Composição químico-bromatológica e percentual dos minerais nas dietas experimentais com base na matéria seca e balanço cátion-aniônico da dieta (BCAD) em mEq de $\left\{(\mathrm{Na}+\mathrm{K})-\left(\mathrm{Cl}+\mathrm{SO}_{4}\right)\right\}$

\begin{tabular}{lccccc}
\hline & $\mathrm{N} 1$ & $\mathrm{~N} 2$ & $\mathrm{C}$ & $\mathrm{P} 1$ & $\mathrm{P} 2$ \\
\hline Proteína bruta (\%) & 19,15 & 20,47 & 18,63 & 20,98 & 19,37 \\
Fibra bruta (\%) & 9,46 & 8,56 & 8,63 & 8,16 & 9,11 \\
Extrato etéreo (\%) & 7,24 & 6,70 & 6,38 & 6,70 & 6,70 \\
Matéria mineral (\%) & 8,52 & 9,10 & 10,99 & 9,73 & 9,64 \\
Cálcio (\%) & 1,11 & 1,11 & 1,10 & 1,12 & 1,13 \\
Fósforo (\%) & 0,55 & 0,55 & 0,55 & 0,55 & 0,55 \\
Sódio (\%) & 0,35 & 0,35 & 0,35 & 0,65 & 0,85 \\
Potássio (\%) & 1,25 & 1,25 & 1,28 & 1,18 & 1,20 \\
Enxofre (\%) & 0,80 & 0,60 & 0,35 & 0,37 & 0,36 \\
Cloro (\%) & 0,46 & 0,43 & 0,44 & 0,40 & 0,40 \\
BCAD & $-159,88$ & $-40,54$ & 138,79 & 251,01 & 500,49 \\
\hline
\end{tabular}

N1, N2, C, P1 e P2 = tratamentos segundo o BCAD.

Os animais, alimentados às 8 e às 14 horas, em $4,4 \%$ do peso vivo, foram pesados a cada 15 dias. Para a realização do estudo de digestibilidade, foram feitas colheitas de fezes e da dieta, na proporção de $10 \%$. No $30^{\circ}$ e no $60^{\circ}$ dias foram retiradas amostras de fluido ruminal através de sonda esofagiana, com análise imediata de $\mathrm{pH}$ e retirada de subamostras, diluídas em proporção de $25 \%$ com ácido fórmico para posteriores análises de ácidos graxos voláteis.

As determinações de matéria seca e matéria mineral nas fezes e rações obedeceram às recomendações do Official... (1990). O sódio foi determinado por fotometria, o enxofre, por turbidimetria em sistema flow injection analysis, e o cloro, por titulometria com nitrato de prata. O fósforo foi analisado colorimetricamente, seguindo a metodologia proposta por Fiske e Subbarow (1925).
$\mathrm{O} \mathrm{pH}$ do líquido ruminal foi mensurado imediatamente após a colheita. As subamostras, contendo $4 \mathrm{ml}$ de líquido ruminal e $1 \mathrm{ml}$ de ácido fórmico, foram descongeladas e centrifugadas a $10^{\circ} \mathrm{C}$ por 20 minutos a 20.000g. As determinações dos ácidos graxos voláteis foram feitas por meio de cromatografia líquida.

No delineamento experimental em blocos ao acaso, foram utilizados 25 animais, com cinco tratamentos e cinco repetições por tratamento. Os dados foram analisados por meio do programa computacional SAS (SAS, 1985). As variáveis foram analisadas no PROC GLM, por regressão, com os níveis do BCAD (-160, -40 140, 250 e $500 \mathrm{mEq} / \mathrm{kg}$ de MS). As variáveis também foram analisadas por contrastes ortogonais por meio do PROC GLM, entre os tratamentos N1(-160 $\mathrm{mEq} / \mathrm{kg}$ de $\mathrm{MS}), \mathrm{N} 2$ (-40 $\mathrm{mEq} / \mathrm{kg}$ de $\mathrm{MS}), \mathrm{C}$ (140 $\mathrm{mEq} / \mathrm{kg}$ de MS), P1(+250 mEq/kg de MS) e $\mathrm{P} 2(+500 \mathrm{mEq} / \mathrm{kg}$ de MS), e também entre os 
tratamentos negativos (N1 e N2), o controle (C) e os positivos (P1 e P2).

\section{RESULTADOS E DISCUSSÃO}

$\mathrm{O}$ dados referentes à ingestão de matéria seca (IMS) são apresentados na Tab. 3, onde se verifica relação linear entre o BCAD e a IMS. Em geral, a IMS aumentou a partir do BCAD de 140 até o de $500 \mathrm{mEq} / \mathrm{kg}$ de MS.

Os resultados assemelham-se aos relatados por Fauchon et al. (1995), os quais, em experimento desenvolvido com cordeiros que receberam BCAD de 100, 300, 500 e $700 \mathrm{mEq}$ de $(\mathrm{Na}+\mathrm{K})-$ (Cl) / $\mathrm{kg}$ de MS por 42 dias, encontraram efeito linear significativo $(\mathrm{P}<0,01)$ sobre a IMS. Os autores calcularam o BCAD apenas com a inclusão de $\mathrm{Cl}$ como ânion e não forneceram os valores de $\mathrm{SO}_{4}$ na dieta. Dessa forma, para efeito de comparação com o presente trabalho, utilizando-se o Nutrient... (1985), foi calculado o novo BCAD, resultando nos seguintes valores: 4 , 175,390 e $580 \mathrm{mEq}(\mathrm{Na}+\mathrm{K})-(\mathrm{Cl}+\mathrm{S}) / \mathrm{kg}$ de MS. A IMS aumentou quando o BCAD variou de 4 a $390 \mathrm{mEq} / \mathrm{kg}$ de MS e não se alterou nos valores superiores a $390 \mathrm{mEq} / \mathrm{kg}$ de MS. Ross et al. (1994) encontraram efeito linear positivo do BCAD com a IMS até o $84^{\circ}$ dia de experimento. Esses autores também não forneceram os valores de $\mathrm{SO}_{4}$ nas dietas experimentais; desse modo, utilizando o mesmo procedimento, os dados foram recalculados, chegando-se aos seguintes valores: -50, 85, 200 e $395 \mathrm{mEq}(\mathrm{Na}+\mathrm{K})-$ $(\mathrm{Cl}+\mathrm{S}) \quad / \mathrm{kg} \mathrm{MS}$, com a ingestão máxima observada no BCAD de $395 \mathrm{mEq} / \mathrm{kg}$ de MS.

Tabela 3. Níveis médios de ingestão de matéria seca, em gramas por $\mathrm{kg}{ }^{0,75}$, em ovinos alimentados com diferentes níveis de balanço cátion-aniônico da dieta (tratamentos) em $\mathrm{mEq} / \mathrm{kg}$ de matéria seca

\begin{tabular}{|c|c|c|c|c|c|c|c|c|c|}
\hline & \multicolumn{5}{|c|}{ Tratamento } & \multicolumn{4}{|c|}{ Análise de variância } \\
\hline & -160 & -40 & 140 & 250 & 500 & CV (\%) & $\mathrm{L}$ & $\mathrm{Q}$ & $\mathrm{C}$ \\
\hline Dias $0-15$ & 74,31 & 76,52 & 71,83 & 75,19 & 77,02 & 3,31 & 0,04 & 0,04 & 0,37 \\
\hline Dias $15-30$ & 75,80 & 81,863 & 75,43 & 81,37 & 83,04 & 4,17 & $<0,01$ & 0,37 & 0,97 \\
\hline Dias $30-45$ & 83,20 & 88,560 & 84,29 & 90,61 & 92,89 & 5,55 & $<0,01$ & 0,57 & 0,59 \\
\hline Dias 45-60 & 93,58 & 98,587 & 93,78 & 102,37 & 102,97 & 5,40 & $<0,01$ & 0,67 & 0,29 \\
\hline Dias 60-75 & 103,32 & 107,04 & 104,33 & 112,98 & 112,98 & 5,93 & $<0,01$ & 0,89 & 0,15 \\
\hline Total & 86,04 & 90,516 & 92,57 & 93,78 & 93,78 & 4,67 & $<0,01$ & 0,50 & 0,34 \\
\hline
\end{tabular}

$\mathrm{CV}=$ coeficiente de variação; $\mathrm{L}=$ efeito linear; $\mathrm{Q}=$ efeito quadrático; $\mathrm{C}=$ efeito cúbico.

Leek e Harding (1975), citados por Forbes (1995), demonstraram a existência de quimioreceptores localizados no rúmen e retículo com atividade dependente do $\mathrm{pH}$, sugerindo que a IMS diminui com a queda do $\mathrm{pH}$ ruminal. A explicação residiria no efeito do $\mathrm{pH}$ na motilidade ruminal. Dessa forma, uma explicação da linearidade entre BCAD e IMS seria a relação existente entre o BCAD e o pH ruminal, principalmente em níveis positivos.

$\mathrm{Na}$ Tab 4 apresentam-se dados referentes à média de ganho de peso diário, à eficiência alimentar e à digestibilidade de matéria seca.

A digestibilidade aparente da matéria seca não foi alterada pelo BCAD. O ganho de peso médio e a eficiência alimentar, durante os 75 dias de experimento, aumentaram de forma linear.

Os resultados são semelhantes aos apresentados por Fauchon et al. (1995), os quais, objetivando estudar a relação entre BCAD e o desempenho em cordeiros, não encontraram diferenças na digestibilidade de matéria seca, entretanto o aumento do BCAD resultou em aumento linear no GPD. Esses autores encontraram o maior GPD no BCAD de $+580 \mathrm{mEq} / \mathrm{kg}$ MS e não alteração da eficiência alimentar. A máxima eficiência encontrada por eles foi 0,170 , diferente do presente experimento, 0,234, o que pode ser reflexo da diferença de peso dos animais do experimento. A digestibilidade de matéria seca também não se modificou em função dos diferentes níveis de BCAD (5 a $580 \mathrm{mEq} / \mathrm{kg}$ de MS), resultado confirmado neste experimento. Esses autores consideraram o BCAD entre 390 e $580[(\mathrm{Na}+\mathrm{K})-(\mathrm{Cl}+\mathrm{S})] / \mathrm{kg}$ de $\mathrm{MS}$ os de melhor resultado no desempenho de cordeiros, semelhante ao nível de BCAD preconizado no presente experimento. 
Tabela 4. Valores médios da digestibilidade aparente de matéria seca (DA), ganho de peso diário em $\mathrm{kg}$ por dia (GPD) e eficiência alimentar em $\mathrm{kg}$ de ganho de peso por $\mathrm{kg}$ de matéria seca ingerida (EA), em ovinos alimentados com diferentes níveis de balanço cátion-aniônico da dieta (tratamentos)

\begin{tabular}{|c|c|c|c|c|c|c|c|c|c|}
\hline \multirow{2}{*}{ Variável } & \multicolumn{5}{|c|}{ Tratamento } & \multicolumn{4}{|c|}{ Análise de variância } \\
\hline & -160 & -40 & 140 & 250 & 500 & CV(\%) & $\mathrm{L}$ & $\mathrm{Q}$ & $\mathrm{C}$ \\
\hline $\begin{array}{l}\text { DA } \\
\text { GPD (kg) }\end{array}$ & 71,06 & 70,83 & 70,23 & 72,25 & 72,76 & 2,88 & 0,12 & 0,38 & 0,52 \\
\hline Dias $0-15$ & 0,03 & 0,095 & 0,062 & 0,110 & 0,107 & 46,67 & $<0,01$ & 0,30 & 0,32 \\
\hline Dias $15-30$ & 0,13 & 0,121 & 0,159 & 0,169 & 0,181 & 33,99 & 0,05 & 0,97 & 0,34 \\
\hline Dias 30-45 & 0,190 & 0,188 & 0,176 & 0,223 & 0,197 & 17,15 & 0,36 & 0,76 & 0,08 \\
\hline Dias 45-60 & 0,186 & 0,164 & 0,204 & 0,217 & 0,197 & 20,73 & 0,16 & 0,46 & 0,07 \\
\hline Dias 60-75 & 0,218 & 0,196 & 0,174 & 0,191 & 0,197 & 24,01 & 0,48 & 0,281 & 0,61 \\
\hline Total & 0,151 & 0,153 & 0,155 & 0,182 & 0,174 & 14,14 & 0,02 & 0,80 & 0,14 \\
\hline EA & & & & & & & & & \\
\hline Dias 0-15 & 0,044 & 0,157 & 0,115 & 0,190 & 0,180 & 48,03 & 0,01 & 0,21 & 0,31 \\
\hline Dias $15-30$ & 0,210 & 0,185 & 0,264 & 0,254 & 0,272 & 36,63 & 0,14 & 0,77 & 0,56 \\
\hline Dias 30-45 & 0,296 & 0,255 & 0,255 & 0,296 & 0,245 & 19,99 & 0,43 & 0,93 & 0,12 \\
\hline Dias 45-60 & 0,236 & 0,190 & 0,250 & 0,241 & 0,221 & 20,02 & 0,70 & 0,56 & 0,10 \\
\hline Dias $60-75$ & 0,245 & 0,213 & 0,195 & 0,195 & 0,193 & 24,00 & 0,05 & 0,34 & 0,94 \\
\hline Total & 0,200 & 0,203 & 0,216 & 0,234 & 0,222 & 10,71 & 0,05 & 0,42 & 0,21 \\
\hline
\end{tabular}

$\mathrm{CV}=$ coeficiente de variação. $\mathrm{L}=$ efeito linear; $\mathrm{Q}=$ efeito quadrático; $\mathrm{C}=$ efeito cúbico. $\mathrm{DA}=$ digestibilidade aparente da matéria seca; $\mathrm{EA}=$ eficiência alimentar (ganho/ingestão).

Jackson et al. (2001), em experimento conduzido com bezerros tratados com 0 ou $200 \mathrm{mEq}$ $[(\mathrm{Na}+\mathrm{K})-(\mathrm{Cl}+\mathrm{S})] / \mathrm{kg}$ de $\mathrm{MS}$, ou seja, fora da faixa de BCAD encontrada no presente experimento para o melhor desempenho em cordeiros em crescimento, não observaram diferenças no GPD médio. Entretanto, Jackson et al. (2001), ao estudarem os efeitos do BCAD no desempenho de bezerros e utilizarem -179, 45, 225 e $383 \mathrm{mEq}[(\mathrm{Na}+\mathrm{K})-(\mathrm{Cl}+\mathrm{S})] / \mathrm{kg}$ de $\mathrm{MS}$, encontraram melhores resultados no tratamento $+225 \mathrm{mEq}[(\mathrm{Na}+\mathrm{K})-\mathrm{Cl}] / \mathrm{kg}$ de $\mathrm{MS}$, o que se confirmou no presente experimento.

Os maiores desempenhos observados para dietas com adição de $\mathrm{NaHCO}_{3}$ (P1 e P2) são explicados pelo aumento na IMS e na EA. Block (1994) sugeriu que a bomba de sódio e potássio, mecanismo que opera juntamente com a entrada de glicose nas células, pode se alterar por desequilíbrio entre esses dois minerais, sendo esse um dos motivos da influência do BCAD no desempenho animal.

A análise de contrastes revelou que os tratamentos de BCAD negativos (N1 e N2) e o tratamento-controle apresentaram médias de GPD inferiores às dos $\mathrm{BCAD}$ positivos $(\mathrm{P} 1 \mathrm{e}$ $\mathrm{P} 2$ ). Como nos processos metabólicos são produzidas substâncias acidogênicas, o efeito antagonístico dos cátions poderia explicar o melhor desempenho dos animais mantidos nas dietas com BCAD positivo.

$\mathrm{Na}$ Tab. 5 encontram-se os valores de $\mathrm{pH}$ do líquido ruminal, referentes ao $30^{\circ}$ e $60^{\circ}$ dias, em função de diferentes níveis de BCAD. Observase que houve efeitos linear e quadrático significativos. $\mathrm{O}$ aumento do BCAD aumentou o pH ruminal. Todos os valores são considerados normais para ruminantes que receberam altos níveis de concentrado e fibra curta, como é o caso da casca de algodão.

Tabela 5.Valores médios de $\mathrm{pH}$ do líquido ruminal no $30^{\circ}$ e $60^{\circ}$ dias de experimento em cordeiros alimentados com diferentes níveis de balanço cátion-aniônico da dieta (tratamentos)

\begin{tabular}{|c|c|c|c|c|c|c|c|c|c|}
\hline & \multicolumn{5}{|c|}{ Tratamento } & \multicolumn{4}{|c|}{ Análise de variância } \\
\hline & -160 & -40 & 140 & 250 & 500 & $\mathrm{CV}(\%)$ & $\mathrm{L}$ & $\mathrm{Q}$ & $\mathrm{C}$ \\
\hline $30^{\circ}$ dia & 6,20 & 6,49 & 6,47 & 6,56 & 6,57 & 2,81 & 0,01 & 0,14 & 0,24 \\
\hline $60^{\circ}$ dia & 5,79 & 6,07 & 6,27 & 6,23 & 6,29 & 2,31 & $<0,01$ & 0,01 & 0,47 \\
\hline Média & 6,00 & 6,24 & 6,37 & 6,42 & 6,40 & 1,89 & $<0,01$ & $<0,01$ & 0,25 \\
\hline
\end{tabular}

$\mathrm{CV}=$ coeficiente de variação; $\mathrm{L}=$ efeito linear; $\mathrm{Q}=$ efeito quadrático; $\mathrm{C}=$ efeito cúbico. 
$\mathrm{O} \mathrm{pH}$ do líquido ruminal reflete o balanço entre as taxas de produção e absorção ruminal dos ácidos graxos voláteis, o tamponamento por meio de substâncias contidas na saliva e a presença de tampões ou bases dos alimentos (Van Soest, 1994). Dietas ricas em concentrado requerem baixo tempo de ruminação, conseqüentemente, reduzem a secreção salivar em relação à produção de ácidos graxos voláteis (Orskov e Ryle, 1990). No presente experimento, a dieta continha $30 \%$ de casca de algodão como fonte volumosa, ou seja, fibra não efetiva, o que explica os valores de $\mathrm{pH}$ ruminal encontrados em todos os tratamentos. O líquido ruminal foi colhido por sonda esofagiana, o que também pode influenciar o $\mathrm{pH}$, pois pode existir contaminação de saliva. Estes resultados são condizentes com os de Tucker et al. (1988), que relataram aumento do $\mathrm{pH}$ do fluido ruminal de forma linear com o aumento nos níveis de BCAD em vacas leiteiras. Para os tratamentos de -100 , 0,100 ou $200 \mathrm{mEq}(\mathrm{Na}+\mathrm{K})-(\mathrm{Cl}+\mathrm{S}) / \mathrm{kg}$ de $\mathrm{MS}$, os valores encontrados foram de 6,5, 6,63, 6,73 e 6,76 , respectivamente. Menores valores de $\mathrm{pH}$ do líquido ruminal para tratamentos com $\mathrm{BCAD}$ negativo também foram observados por Vagnoni e OetzeL (1998), os quais trabalharam com vacas secas e diferentes sais aniônicos, com BCAD de $203,-51,-40$ e $-63 \mathrm{mEq}(\mathrm{Na}+\mathrm{K})-(\mathrm{Cl}+\mathrm{S}) / \mathrm{kg}$ de MS. Esses autores verificaram queda de 0,12 unidades de $\mathrm{pH}$ quando as vacas foram alimentadas com sais aniônicos em relação ao período de alimentação basal (203 $\mathrm{mEq}(\mathrm{Na}+\mathrm{K})$ $(\mathrm{Cl}+\mathrm{S}) / \mathrm{kg})$. Os resultados deste estudo estão de acordo com os relatados por Ross et al. (1994), em experimento desenvolvido com novilhos em crescimento, com tratamentos de BCAD -50, 85, 200 e $395 \mathrm{mEq}(\mathrm{Na}+\mathrm{K})-(\mathrm{Cl}+\mathrm{S})] / \mathrm{kg}$ MS. Esses autores, com a colheita de fluido ruminal por sonda esofagiana, verificaram efeito linear no $28^{\circ}$ dia de experimento. $\mathrm{O} \mathrm{pH}$ aumentou de 6,66 no BCAD de -50 até 6,93 no BCAD de 200 , permanecendo inalterado até o BCAD de 395 $\mathrm{mEq}(\mathrm{Na}+\mathrm{K})-(\mathrm{Cl}+\mathrm{S})] / \mathrm{kg} \mathrm{MS}$.

Os menores valores encontrados para as dietas negativas, provavelmente, são resultado de aumento do strong ion difference (SID), no fluido ruminal. Stewart (1983) afirmou que a concentração do íon de hidrogênio é dependente da diferença entre as concentrações de cátions fortes e de ânions fortes em uma solução (SID). Conseqüentemente, mudança no $\mathrm{pH}$ indica que deve haver mudança em uma dessas variáveis independentes. $\mathrm{O}$ SID é definido pela concentração de $(\mathrm{Na}+\mathrm{K})-\left(\mathrm{Cl}+\mathrm{SO}_{4}\right)$. O $\left(\mathrm{NH}_{4}\right)_{2} \mathrm{SO}_{4}$ encontrado nos tratamentos de -160 e $-40 \mathrm{mEq} / \mathrm{kg}$ de MS diminui o SID no rúmen, pela alta concentração de $\mathrm{SO}_{4}$, provavelmente diminuindo o $\mathrm{pH}$ desse fluido. A mesma explicação é válida para o maior $\mathrm{pH}$ com a inclusão de $\mathrm{NaHCO}_{3}$ (+250 e $500 \mathrm{mEq} / \mathrm{kg}$ de MS). Os tratamentos com adição de $\mathrm{NaHCO}_{3}$ ainda aumentam os valores do $\mathrm{pH}$ por tamponamento do bicarbonato, por aumento da ingestão de água, por diluição do conteúdo ruminal e, conseqüentemente, por aumento de fluxo (Russel e Chow, 1993). Dessa forma, a relação positiva encontrada entre $\mathrm{pH}$ ruminal e BCAD, provavelmente, é conseqüência de alteração no SID no rúmen e também por tamponamento dos ácidos produzidos, nos tratamentos com a inclusão de $\mathrm{NaHCO}_{3}$.

Os resultados de fermentação ruminal, analisada pelo perfil de ácidos graxos voláteis, são apresentados nas Tab. 6 e 7. Foram realizadas colheitas nos dias 30 e 60 do período experimental, através de sonda esofagiana.

Tabela 6. Concentrações médias de ácidos graxos voláteis em $\mathrm{mMol} / \mathrm{dl}$ e em percentual do total no $30^{\circ}$ dia, em ovinos alimentados com diferentes níveis de balanço cátion-aniônico da dieta (tratamentos)

\begin{tabular}{|c|c|c|c|c|c|c|c|c|c|}
\hline & & & ratamen & & & & álise & ariânc & \\
\hline & -160 & -40 & 140 & 250 & 500 & $\mathrm{CV}(\%)$ & $\mathrm{L}$ & $\mathrm{Q}$ & $\mathrm{C}$ \\
\hline Ácido acético & 27,67 & 30,91 & 26,76 & 22,95 & 31,08 & 26,28 & 0,92 & 0,37 & 0,20 \\
\hline Ácido acético $(\%)^{1}$ & 66,09 & 62,63 & 64,60 & 61,99 & 63,41 & 11,43 & 0,70 & 0,63 & 0,57 \\
\hline Ácido propiônico & 10,93 & 16,00 & 11,67 & 12,92 & 15,97 & 47,55 & 0,54 & 0,94 & 0,19 \\
\hline Ácido propiônico (\%) & 26,23 & 31,54 & 29,15 & 30,83 & 30,73 & 27,40 & 0,61 & 0,63 & 0,38 \\
\hline Ácido butírico & 3,19 & 2,55 & 2,53 & 2,88 & 2,893 & 41,21 & 0,81 & 0,64 & 0,75 \\
\hline Ácido butírico $(\%)^{1}$ & 7,66 & 5,83 & 6,25 & 7,17 & 5,85 & 29,75 & 0,48 & 0,85 & 0,14 \\
\hline $\mathrm{A} / \mathrm{P}^{*}$ & 2,71 & 2,120 & 2,31 & 2,17 & 2,554 & 45,43 & 0,89 & 0,31 & 0,52 \\
\hline Total & 41,74 & 49,80 & 40,96 & 38,75 & 49,95 & 28,49 & 0,72 & 0,55 & 0,17 \\
\hline
\end{tabular}


Tabela 7. Concentrações médias de ácidos graxos voláteis em $\mathrm{mMol} / \mathrm{dl}$ e em percentual do total no $60^{\circ}$ dia, em ovinos alimentados com diferentes níveis de balanço cátion-aniônico da dieta (tratamentos)

\begin{tabular}{|c|c|c|c|c|c|c|c|c|c|}
\hline & \multicolumn{5}{|c|}{ Tratamento } & \multicolumn{4}{|c|}{ Análise de variância } \\
\hline & -160 & -40 & 140 & 250 & 500 & $\mathrm{CV}(\%)$ & $\mathrm{L}$ & $\mathrm{Q}$ & $\mathrm{C}$ \\
\hline Ácido acético & 19,61 & 22,75 & 31,75 & 26,79 & 22,83 & 23,94 & 0,21 & $<0,01$ & 0,93 \\
\hline Ácido acético $(\%)^{1}$ & 71,22 & 65,76 & 72,31 & 60,44 & 68,24 & 8,57 & 0,35 & 0,25 & 0,55 \\
\hline Ácido propiônico & 5,65 & 9,59 & 9,68 & 16,10 & 9,01 & 59,56 & 0,23 & 0,03 & 0,48 \\
\hline Ácido propiônico $(\%)^{1}$ & 21,64 & 27,30 & 21,63 & 34,54 & 25,86 & 21,40 & 0,21 & 0,27 & 0,74 \\
\hline Ácido butírico & 1,92 & 2,45 & 2,72 & 3,71 & 1,96 & 45,64 & 0,63 & 0,03 & 0,33 \\
\hline Ácido butírico $(\%)^{1}$ & 7,130 & 6,93 & 6,052 & 7,71 & 5,89 & 22,95 & 0,33 & 0,63 & 0,29 \\
\hline $\mathrm{A} / \mathrm{P}^{*}$ & 3,36 & 2,56 & 3,44 & 2,02 & 2,79 & 26,28 & 0,25 & 0,35 & 0,83 \\
\hline Total & 26,59 & 34,79 & 44,17 & 46,61 & 38,81 & 32,22 & 0,21 & 0,01 & 0,68 \\
\hline
\end{tabular}

$\mathrm{CV}$ = coeficiente de variação; $\mathrm{L}=$ efeito linear; $\mathrm{Q}=$ efeito quadrático; $\mathrm{C}=$ efeito cúbico. $* \mathrm{~A} / \mathrm{P}$ - acético/propiônico. ${ }^{1} \%$ em relação ao total.

De maneira geral, tanto no $30^{\circ}$ quanto no $60^{\circ}$ dia de experimento, o $\mathrm{BCAD}$ não influenciou $\mathrm{o}$ perfil de ácidos graxos voláteis no rúmen. Apenas no $30^{\circ}$ dia de experimento, a produção de ácido acético, em mMol por $\mathrm{dl}$, apresentou comportamento quadrático, aumentando até o BCAD de 140 e diminuindo posteriormente até o BCAD de $500 \mathrm{mEq} / \mathrm{kg}$ de MS. Também no $30^{\circ}$ dia, a concentração de ácido butírico e a concentração total de ácidos graxos voláteis apresentaram comportamento quadrático, aumentando até o BCAD de 250 e diminuindo no de $500 \mathrm{mEq} / \mathrm{kg}$ de $\mathrm{MS}$, podendo ser reflexo da diluição ruminal em função do maior consumo de água.

Esses resultados são compatíveis com os observados por Tucker et al. (1988), os quais, em experimento conduzido com vacas lactantes, tratadas com BCAD de -100 até $+200 \mathrm{mEq} / \mathrm{kg}$ de MS, concluíram que o BCAD não interfere no perfil de AGVs, embora o total de AGV tendesse a diminuir com o aumento do BCAD. Ross et al. (1994) também argumentaram que o BCAD tem pouca influência nas proporções molares de AGVs. Os autores verificaram crescimento linear do ácido butírico e propiônico no $84^{\circ}$ dia de experimento. Vagnoni e Oetzel (1998), em experimento comparando sais aniônicos, também não encontraram efeitos do $\mathrm{BCAD}$ na proporção individual de AGVs, ou na relação entre acético e propiônico, e sugeriram que esses sais exerceriam modesto efeito na fermentação ruminal. Pelos resultados obtidos e pela literatura consultada, o BCAD tem pouca influência no perfil de ácidos graxos voláteis no rúmen.

\section{CONCLUSÕES}

Conclui-se que o balanço cátion-aniônico da dieta influencia o desempenho, pelo aumento da ingestão de matéria seca, o ganho de peso diário e a eficiência alimentar nas dietas catiônicas de ovinos da raça Santa Inês.

\section{REFERÊNCIAS BIBLIOGRÁFICAS}

BLOCK, E. Manipulation of dietary cation-anion difference on nutritionally reted production diseases, productivity, and metabolic responses of dairy cows. J. Dairy Sci., v.77, p.1437-1450, 1994.

CUNNINGHAM, J.G. Tratado de fisiologia veterinária. Rio de Janeiro: Guanabara, 1999. $454 \mathrm{p}$.

DEN HARTOG, L.A.; MORS, R.A.B.; VERSTEGEN, M.W.A. et al. The dietary electrolyte balance in oral calf nutrition. J. Anim. Physiol. Anim. Nutr., v.63, p.173-179, 1990.

FAUCHON, C.; SEOANE, J.R.; BERNIER, J.F. Effects of dietary cation-anion concentrations on performance and acid-base balance in growing lambs. Can. J. Anim. Sci., v.75, p.145-151, 1995.

FISKE, C.M.; SUBBAROW, Y. The colorimetric determination of phosphorus. $J$. Biol. Chem., v.66, p.375-400, 1925.

FORBES, J.M. Voluntary food intake and diet selection in farm animals. Wallingford: CAB International, 1995. 532p. 
JACKSON, J.A.; AKAY, V.; FRANKLIN, S.T. et al. The effect of cation-anion differnce on calcium requeriment, feed intake, body weight gain, and blood gassas and mineral concentration of dairy calves. J. Dairy Sci., v.84, p.147-153, 2001.

MILES, R.; BUTCHER, G. Equilíbrio ácidobase I : princípios básicos. Ind. Avíc., v.40, p.2124, 1993.

NUTRIENT requeriment of sheep. Washington, DC:NAC, 1985.

OFFICIAL methods of analysis. 15.ed. Arlington:AOAC, 1990. 1298p.

ORSKOV, E.R.; RYLE, M. Energy nutrition in ruminants. New York: Elsevier Applied Science, 1990. 149p.

OWENS, F.N.; SECRIST, D.S.; HILL, W.J. et al. Acidosis in cattle: a review. J. Anim. Sci, v.75, p.727-735, 1998.

ROSS, J. G.; SPEARS, J. W.; GARLICH, J. D. Dietary electrolyte balance effects on performance and metabolic characteristicas in growing steers. J. Anim. Sci., v.72, p.1842-1848,
1994.

RUSSELL, J.B.; CHOW, J.M. Another theory for the action of ruminal buffers salts: decreased fermentation and propionate production. J. Dairy Sci., v.76, p.826-830, 1993.

SAS Stat Guide, Release 6.03 Edition. Cary, NC: SAS Institute, 1988. 1028p.

STEWART, P.A. Modern quantitative acid-base chemistry. Ann. J. Physiol. Pharm., v.61, p.14441461, 1983.

TUCKER, W.B.; XIN, Z.; HEMKEN, R.W. Influence of dietary calcium choride on adaptative changes in acid-base satatus and mineralmetabolism in lactation dairy cows fed a diet hight in sodium bicarbonate. J. Dairy Sci., v.71, p.1587-1597, 1988.

VAGNONI, D.B.; OETZEL, G.R. Effects of dietary cation-anion differnce on the acid-base status of dry cows. J. Dairy Sci., v.81,p.16431652, 1998.

VAN SOEST, P.J. Nutricional ecology of the ruminant. New York: Cornell University, 1994. 476p. 